\title{
Analysis of gene expression patterns in triple negative breast cancer II: TNBC molecular subtype.
}

Shahan Mamoor, MS ${ }^{1}$

${ }^{1}$ shahanmamoor@gmail.com

East Islip, NY 11730

Triple negative breast cancer (TNBC) shares overlap with the basal molecular subtype of breast cancer and is more frequently diagnosed in African-American (black) women for reasons not understood $(1,2)$. To understand genes whose expression may be of pertinence to the development or progression of triple negative breast cancer, we mined published microarray data (3) comparing global gene expression profiles of TNBC molecular subtypes, identifying genes whose expression changed the least between among TNBCs, suggesting that these genes may be important for TNBC biology. We identified PVT1, PHC3, WNT8B, MLLT6, MSH3, IHH, and WNT2B among the genes whose expression differed the least when comparing TNBC cases and subtypes. Kaplan-Meier survival analysis revealed that expression levels of each of these genes correlated with survival outcomes in human breast cancer; in some cases, this correlation differed based on race of the patient, and in other cases, this correlation was found in the basal subtype of human breast cancer, which shares significant overlap with triple negative breast cancer at the level of gene expression (2). PVT1, PHC3, WNT8B, MLLT6, MSH3, IHH, and WNT2B may be of relevance in understanding the etiology or progression of triple negative breast cancer.

Keywords: triple negative breast cancer, breast cancer, PVT, PHC3, WNT2B, MLLT6, WNT8B, IHH, MSH3, systems biology of breast cancer, targeted therapeutics in breast cancer. 
It is not understood why African-American women are more frequently diagnosed with triple negative breast cancer, a disease marked by lack of expression of receptors for the hormones estrogen and progesterone, and for the human epidermal growth factor receptor 2, HER2 (1,2). TNBC shares overlap with the basal-like subtype of breast cancer which is characterized by relatively worse patient outcomes as compared to other subtypes (1). We utilized a transcriptome-wide gene expression analysis approach, mining published microarray data (3) for the discovery of genes with potential relevance to the biology of TNBC, aiming to utilize these findings as a starting point for further exploration and validation. We report here further findings of this analysis.

\section{Methods}

We utilized microarray datasets GSE76124 (3) for this differential gene expression analysis of triple negative breast cancer, in conjunction with GEO2R. GSE76124 was generated using Affymetrix Human Genome U133 Plus 2.0 Array technology with TNBC tumors of four molecular subtypes (TNBC molecular subtypes, different from molecular subtypes basal, luminal A, luminal B, and HER2+): $n=54$ basal-like immune-activated (BLIA), $n=60$ basal-like immune-suppresed (BLIS), $n=37$ luminal-AR (LAR), and $n=47$ mesenchymal (MES); analysis was performed using platform GPL570. The Benjamini and Hochberg method of $\mathrm{p}$-value adjustment was used for ranking of differential expression but raw $p$-values were used to assess statistical significance of global differential expression. Log-transformation of data was auto-detected, and the NCBI generated category of platform annotation was used. For Kaplan-Meier survival analysis, we utilized the Kaplan-Meier survival analysis online tool $(4,5)$, comparing breast cancer cases based on race and evaluating correlations with survival outcomes based on expression of PVT1 or PHC3 in $n=1090$ breast cancer patients, or based on expression of MLLT6, WNT2B, WNT8B, MSH3, or IHH in patients of the basal molecular subtype for recurrence-free survival in $n=417$ or $n=846$ patients, or overall survival in $n=404$ patients.

\section{Results}

We mined published microarray data (3) generated using tumor tissues from patients with triple negative breast cancer (TNBC) for the discovery of genes with importance to the biology of TNBC.

PVT1, PHC3, MLLT6, WNT8B, MSH3. IHH, and WNT2B are among the genes whose expression changes the least transcriptome-wide when comparing TNBC cases based on TNBC molecular subtype

We compared the global gene expression profiles of 198 cases of triple negative breast cancer, divided into four groups based on TNBC molecular subtype: basal-like immune-activated (BLIA), basal-like immune-suppressed (BLIS), luminal-AR (LAR), and mesenchymal (MES), using published microarray data (3). Differential gene expression analyses operate under the principle that genes whose expression differs most between groups compared may be of importance to biological description as they inform the investigator as to the identity of the sample. In this case, we were interested in the opposite: what genes were among those whose expression changed the least? This would direct us towards genes whose importance was shared among TNBC cases - genes that inform the investigator as to the identity of TNBC tumors, regardless of molecular subtype. The expression of 54,675 transcripts in total was compared between four TNBC molecular subtypes. To further refine our selection approach, we sorted through the $0.5 \%$ of transcripts whose expression was least different transcriptome-wide, then asking if any of these genes had previously been reported as important for breast cancer tumorigenesis or invasiveness, or in such a process in another solid tumor type, or have been found to be genetically or transcriptionally perturbed in some breast cancer subtype or cell line. Using this approach, we identified seven genes of interest: PVT1, PHC3, MLLT6, WNT8B, MSH3, IHH, and WNT2B. 
PVT1 differential expression ranked $0.16 \%$ transcriptome-wide, with differential expression at 54585 out of 54675 total transcripts when triple negative breast cancers were compared based on TNBC molecular subtype. Differential expression of PVT1 was not statistically significant (Chart 1; $p=9.95 \mathrm{E}-013633$ ), thus indicating that expression of PVT1 is not significantly different between TNBC cases when compared based on TNBC molecular subtype. PVT1 has been found to be amplified in human breast cancer and function as a non-coding regulator of triple negative breast cancer $(6,7)$.

PHC3 differential expression ranked $0.06 \%$ transcriptome-wide, with differential expression at 54641 out of 54675 total transcripts when triple negative breast cancers were compared based on TNBC molecular subtype. Differential expression of PHC3 was not statistically significant (Chart 2; $p=9.98 \mathrm{E}-01$ ), thus indicating that expression of PHC3 is not significantly different between TNBC cases when compared based on TNBC molecular subtype. PHC3 is amplified in lung (squamous cell carcinomas) and ovarian cancers (8).

MLLT6 differential expression ranked $0.11 \%$ transcriptome-wide, with differential expression at 54614 out of 54675 total transcripts when triple negative breast cancers were compared based on TNBC molecular subtype. Differential expression of MLLT6 was not statistically significant (Chart 3; $p=9.96 \mathrm{E}-01$ ), thus indicating that expression of MLLT6 is not significantly different between TNBC cases when compared based on TNBC molecular subtype. MLLT6 is highly expressed in multiple breast cancer cell lines that have gain or amplification at the ERBB2 locus (9).

WNT8B differential expression ranked $0.02 \%$ transcriptome-wide, with differential expression at 54664 out of 54675 total transcripts when triple negative breast cancers were compared based on TNBC molecular subtype. Differential expression of WNT8B was not statistically significant (Chart 4; $p=9.99 \mathrm{E}-01$ ), thus indicating that expression of WNT8B is not significantly different between TNBC cases when compared based on TNBC molecular subtype. WNT8B message is $\beta$-estradiol-inducible in MCF-7 breast cancer cells, and WNT8B has been reported to support cell proliferation of hepatocellular carcinomas $(10,11)$.

MSH3 differential expression ranked $0.08 \%$ transcriptome-wide, with differential expression at 54629 out of 54675 total transcripts when triple negative breast cancers were compared based on TNBC molecular subtype. Differential expression of MSH3 was not statistically significant (Chart 5; $p=9.97 \mathrm{E}-01$ ), thus indicating that expression of MSH3 is not significantly different between TNBC cases when compared based on TNBC molecular subtype. A single-nucleotide polymorphism in MSH3, rs26279, has been demonstrated to increase the risk of development of multiple cancer types including breast cancer, and germline mutations in MSH3 have been found by exome sequencing in association with colorectal adenomatous polyposis, a familial cancer syndrome $(12,13)$.

IHH differential expression ranked $0.5 \%$ transcriptome-wide, with differential expression at 54404 out of 54675 total transcripts when triple negative breast cancers were compared based on TNBC molecular subtype. Differential expression of IHH was not statistically significant (Chart $6 ; p=9.83 \mathrm{E}-01$ ), thus indicating that expression of IHH is not significantly different between TNBC cases when compared based on TNBC molecular subtype. In a Pakistani cohort, IHH levels were found to be significantly reduced in breast tumor tissues as compared to normal mammary tissue, and a mechanistic study described Runx2 activation of IHH to be involved in processes underlying bone metastasis $(14,15)$.

WNT2B differential expression ranked $0.49 \%$ transcriptome-wide, with differential expression at 54407 out of 54675 total transcripts when triple negative breast cancers were compared based on TNBC molecular subtype. Differential expression of WNT2B was not statistically significant (Chart 7; $p=9.83 \mathrm{E}-01$ ), thus indicating that expression of WNT2B is not significantly different between TNBC cases when compared based on TNBC molecular subtype. Silencing of WNT2B has been reported to impair 
metastasis in an experimental model of ovarian cancer, suggesting WNT2B may be important for solid tumor progression in at least one cancer type (16).

Thus, among genes whose expression is most quantitatively similar, transcriptome-wide, when comparing triple negative breast cancers based on TNBC subtype, include PVT1, PHC3, MLLT6, WNT8B, MSH3, IHH, and WNT2B.

PVT1 and PHC3 primary tumor expression is correlated with survival outcomes in human breast cancer, and this differs based on race.

We performed Kaplan-Meier survival analysis of $n=1090$ breast cancer patients in total to determine whether primary tumor expression of any genes identified through the aforementioned analysis were correlated with survival outcomes in human breast cancer, and whether any such correlation differed based on race.

We observed significant correlation of PVT1 primary tumor expression with recurrence-free survival in black breast cancer patients but not in white breast cancer patients. (Figure 1; $\log$ rank $p$-value: 0.038 for recurrence-free survival in black breast cancer patients, hazard ratio: 2.79 (1.01-7.68) (Fig. 1)). PVT1 mRNA levels were a negative prognostic indicator in white breast cancer patients. PVT1 primary tumor expression was not correlated with recurrence-free survival in white breast cancer patients ((Figure 1 ; $\log$ rank $p$-value: 0.26 for recurrence-free survival in white breast cancer patients, hazard ratio: 0.74 (0.44-1.25) (Fig. 1)).

We also observed correlation of PHC3 primary tumor expression with recurrence-free survival in black breast cancer patients which trended towards statistical significance, but not in white breast cancer patients (Figure 2; log rank $p$-value: 0.08 for recurrence-free survival in black breast cancer patients, hazard ratio: 0.45 (0.18-1.13) (Fig. 2)). PHC3 mRNA levels were a positive prognostic indicator in white breast cancer patients. $\mathrm{PHC} 3$ primary tumor expression was not correlated with recurrence-free survival in white breast cancer patients ((Figure 2; $\log$ rank $p$-value: 0.46 for recurrence-free survival in white breast cancer patients, hazard ratio: 0.82 (0.49-1.38) (Fig. 1)).

MLLT6, WNT8B, MSH3, IHH, and WNT8B primary tumor expression is correlated with patient survival outcomes in the basal subtype of human breast cancer.

Further analysis of Kaplan-Meier data revealed that primary tumor expression of several genes identified in the aforementioned analysis correlated with patient survival outcomes, either recurrence-free survival or overall survival, in patients diagnosed with the basal molecular subtype of human breast cancer which shares significant overlap with triple negative breast cancer (2).

MLLT6 primary tumor expression was significantly correlated with recurrence-free survival (RFS) in patients with basal subtype human breast cancer ((Figure 3; log rank $p$-value: 0.00039 for RFS in basal type breast cancer patients, hazard ratio: 0.57 (0.42-0.78) (Fig. 3)). MLLT6 mRNA levels were a positive prognostic indicator in basal subtype breast cancer patients. Median RFS was 15 months for basal subtype patients with low tumor expression of MLLT6 as compared to basal subtype patients with high tumor expression of MLLT6, who possessed median RFS of 29 months (Chart 8).

WNT8B primary tumor expression was significantly correlated with overall survival (OS) in patients with basal subtype human breast cancer ((Figure 4; log rank $p$-value: 0.017 for OS in basal type breast cancer patients, hazard ratio: 1.58 (1.08-2.31) (Fig. 4)). WNT8B mRNA levels were a negative prognostic indicator in basal subtype breast cancer patients. Median OS was 95.08 months for basal 
subtype patients with low tumor expression of WNT8B as compared to basal subtype patients with high tumor expression of WNT8B, who possessed median OS of 52.92 months (Chart 9).

MSH3 primary tumor expression was significantly correlated with overall survival (OS) in patients with basal subtype human breast cancer, in the lower survival tertile ((Figure 5; log rank $p$-value: 0.035 for OS in basal type breast cancer patients, hazard ratio: 1.58 (1.09-2.44) (Fig. 5)). MSH3 mRNA levels were a negative prognostic indicator in basal subtype breast cancer patients. Median OS was 115 months for basal subtype patients with low tumor expression of MSH3 as compared to basal subtype patients with high tumor expression of MSH3, who possessed median OS of 54.4 months (Chart 10).

IHH primary tumor expression was significantly correlated with overall survival (OS) in patients with basal subtype human breast cancer, in the upper survival quartile ((Figure 6; log rank $p$-value: 0.064 for OS in basal type breast cancer patients, hazard ratio: 1.47 (0.98-2.22) (Fig. 6)). IHH mRNA levels were a negative prognostic indicator in basal subtype breast cancer patients. Median OS was 68.04 type patients with high tumor expression of IHH, who possessed median OS of 40.96 months (Chart 11).

WNT2B primary tumor expression was significantly correlated with recurrence-free survival (RFS) in patients with basal subtype human breast cancer ((Figure 7; $\log$ rank $p$-value: 0.016 for RFS in basal type breast cancer patients, hazard ratio: 0.76 (0.61-0.95) (Fig. 7)). WNT2B mRNA levels were a positive prognostic indicator in basal subtype breast cancer patients. Median RFS was 19 months for basal subtype patients with low tumor expression of WNT2B as compared to basal subtype patients with high tumor expression of WNT2B, who possessed median RFS of 30 months (Chart 12).

Thus, expression of PVT1, PHC3, MLLT6, WNT8B, MSH3, IHH, and WNT2B is, transcriptome-wide, among the most similar in triple negative breast cancers when comparing cases based on molecular subtype, and primary tumor expression of PVT1, PHC3, MLLT6, WNT8B, MSH3, IHH, and WNT2B is correlated with survival outcomes in human breast cancer; in some cases, this correlation differs based on race of the patient, and in other cases, this correlation can be observed in patients diagnosed with the basal-subtype of human breast cancer, which shares significant similarity with triple negative breast cancer. PVT1, PHC3, MLLT6, WNT8B, MSH3, IHH and WNT2B are candidate genes of relevance for understanding the biology of triple negative breast cancer.

\section{Discussion}

We found here through unbiased, systems-level analysis of published tumor transcriptome data that among the genes whose expression is most similar between cases of triple negative breast cancer when compared by TNBC molecular subtype include PVT1, PHC3, MLLT6, WNT8B, MSH3, IHH, and WNT2B. The identification of each of these genes as potential contributors at the genetic level to the etiology or progression of triple negative breast cancer is supported by findings in the scientific literature that suggest that each of these factors may play a role in breast cancer tumorigenesis or invasiveness, or in such a process in another solid tumor type, or have been found to be genetically or transcriptionally perturbed in some breast cancer subtype or cell line. PVT1, PHC3, MLLT6, WNT8B, MSH3, IHH, and WNT2B are candidate genes of interest for understanding the biology of triple negative breast cancer, and understanding sequence variation (single-nucleotide polymorphism and copy number variation) in PVT1, PHC3, MLLT6, WNT8B, MSH3, IHH, and WNT2B in the black and white population may potentially shed light on why TNBC is more prevalent in black females. 


\section{References}

1. Foulkes, W.D., Smith, I.E. and Reis-Filho, J.S., 2010. Triple-negative breast cancer. New England journal of medicine, 363(20), pp.1938-1948.

2. Dietze, E.C., Sistrunk, C., Miranda-Carboni, G., O'regan, R. and Seewaldt, V.L., 2015.

Triple-negative breast cancer in African-American women: disparities versus biology. Nature Reviews Cancer, 15(4), pp.248-254.

3. Burstein, M.D., Tsimelzon, A., Poage, G.M., Covington, K.R., Contreras, A., Fuqua, S.A., Savage, M.I., Osborne, C.K., Hilsenbeck, S.G., Chang, J.C. and Mills, G.B., 2015. Comprehensive genomic analysis identifies novel subtypes and targets of triple-negative breast cancer. Clinical Cancer Research, 21(7), pp.1688-1698

4. Nagy, Á., Munkácsy, G. and Győrffy, B., 2021. Pancancer survival analysis of cancer hallmark genes. Scientific reports, 11(1), pp.1-10.

5. Györffy, B., Lanczky, A., Eklund, A.C., Denkert, C., Budczies, J., Li, Q. and Szallasi, Z., 2010. An online survival analysis tool to rapidly assess the effect of 22,277 genes on breast cancer prognosis using microarray data of 1,809 patients. Breast cancer research and treatment, 123(3), pp.725-731.

6. Guan, Y., Kuo, W.L., Stilwell, J.L., Takano, H., Lapuk, A.V., Fridlyand, J., Mao, J.H., Yu, M., Miller, M.A., Santos, J.L. and Kalloger, S.E., 2007. Amplification of PVT1 contributes to the pathophysiology of ovarian and breast cancer. Clinical cancer research, 13(19), pp.5745-5755.

7. Tang, J., Li, Y., Sang, Y., Yu, B., Lv, D., Zhang, W. and Feng, H., 2018. LncRNA PVT1 regulates triple-negative breast cancer through KLF5/beta-catenin signaling. Oncogene, 37(34), pp.4723-4734.

8. Crea, F., Sun, L., Pikor, L., Frumento, P., Lam, W.L. and Helgason, C.D., 2013. Mutational analysis of Polycomb genes in solid tumours identifies PHC3 amplification as a possible cancer-driving genetic alteration. British journal of cancer, 109(6), pp.1699-1702.

9. Kauraniemi, P., Bärlund, M., Monni, O. and Kallioniemi, A., 2001. New amplified and highly expressed genes discovered in the ERBB2 amplicon in breast cancer by cDNA microarrays. Cancer research, 61(22), pp.8235-8240.

10. Saitoh, T., Mine, T. and Katoh, M., 2002. Expression and regulation of WNT8A and WNT8B mRNAs in human tumor cell lines: Up-regulation of WNT8B mRNA by $\beta$-estradiol in MCF-7 cells, and down-regulation of WNT8A and WNT8B mRNAs by retinoic acid in NT2 cells. International journal of oncology, 20(5), pp.999-1003.

11. Liu, Y., Di Wu, H.C., Chen, L., Zhang, W., Zou, L., Gao, Q., Zhao, Z., Chen, Q., Zeng, W., Zhang, Z. and Jiang, W., 2021. Wnt8B, transcriptionally regulated by ZNF191, promotes cell proliferation of hepatocellular carcinoma via Wnt signaling. Cancer science, 112(2), p.629.

12. Miao, H.K., Chen, L.P., Cai, D.P., Kong, W.J., Xiao, L. and Lin, J., 2015. MSH3 rs26279 pathology, 8(9), p.11060. 


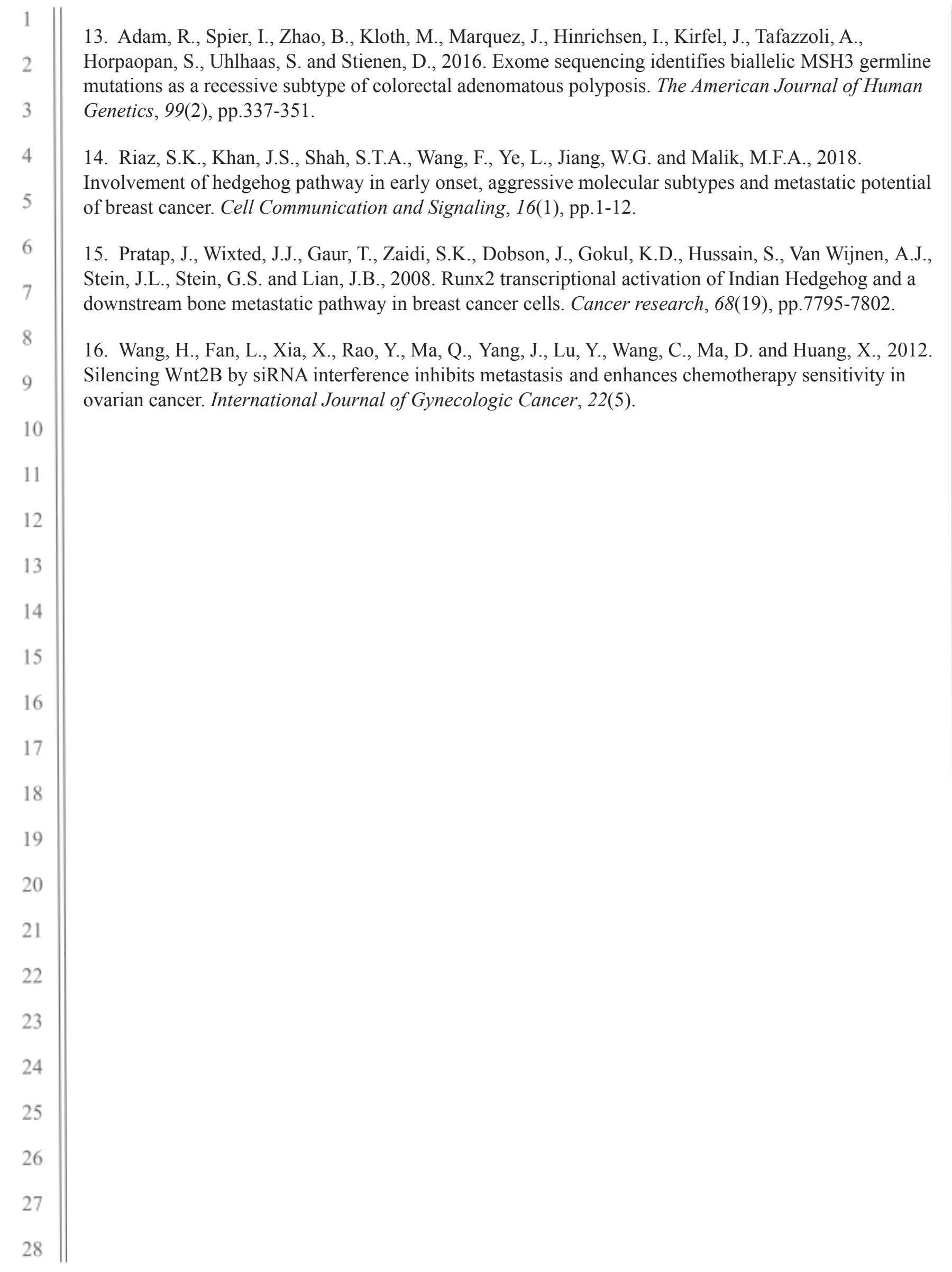




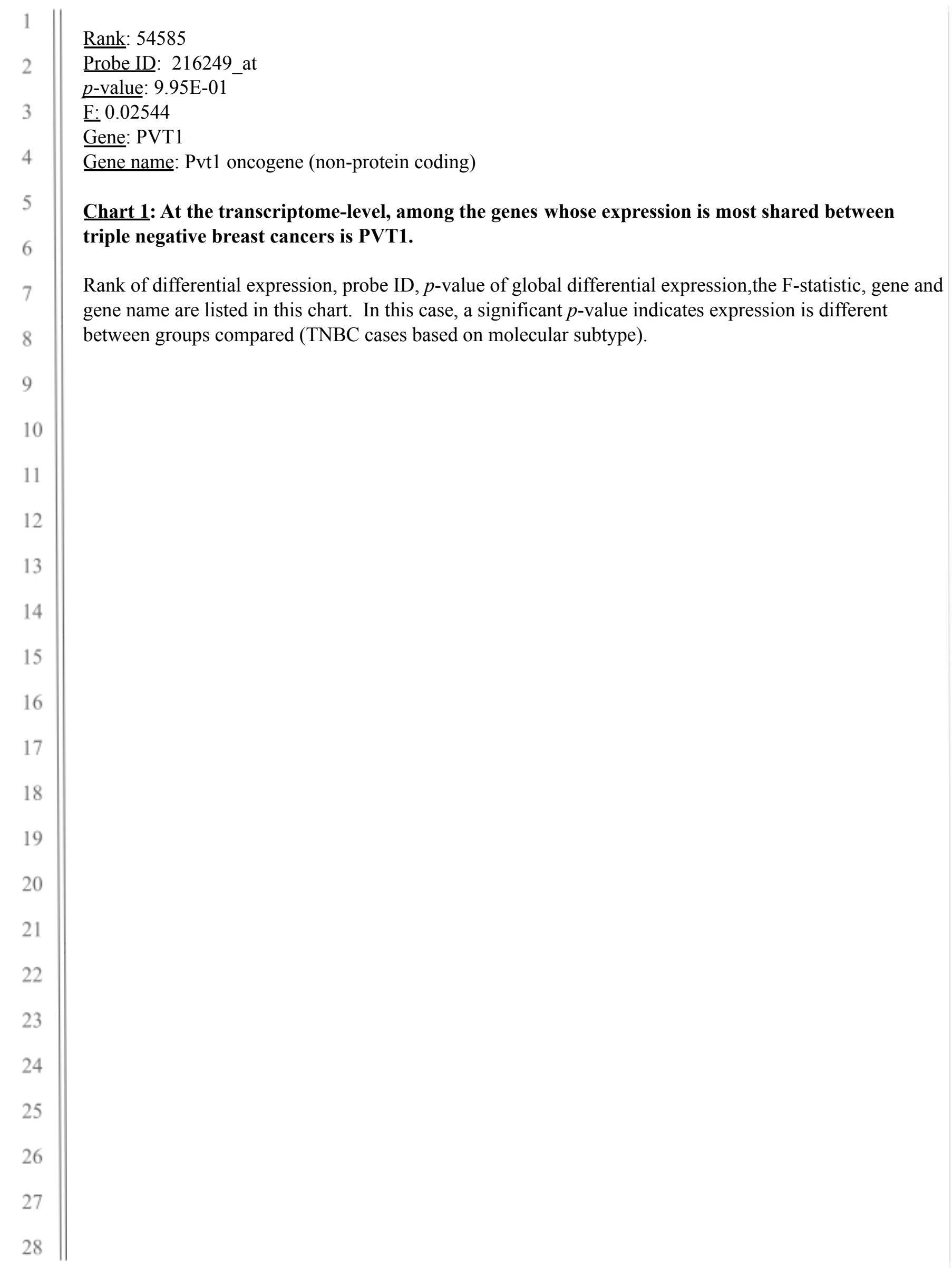




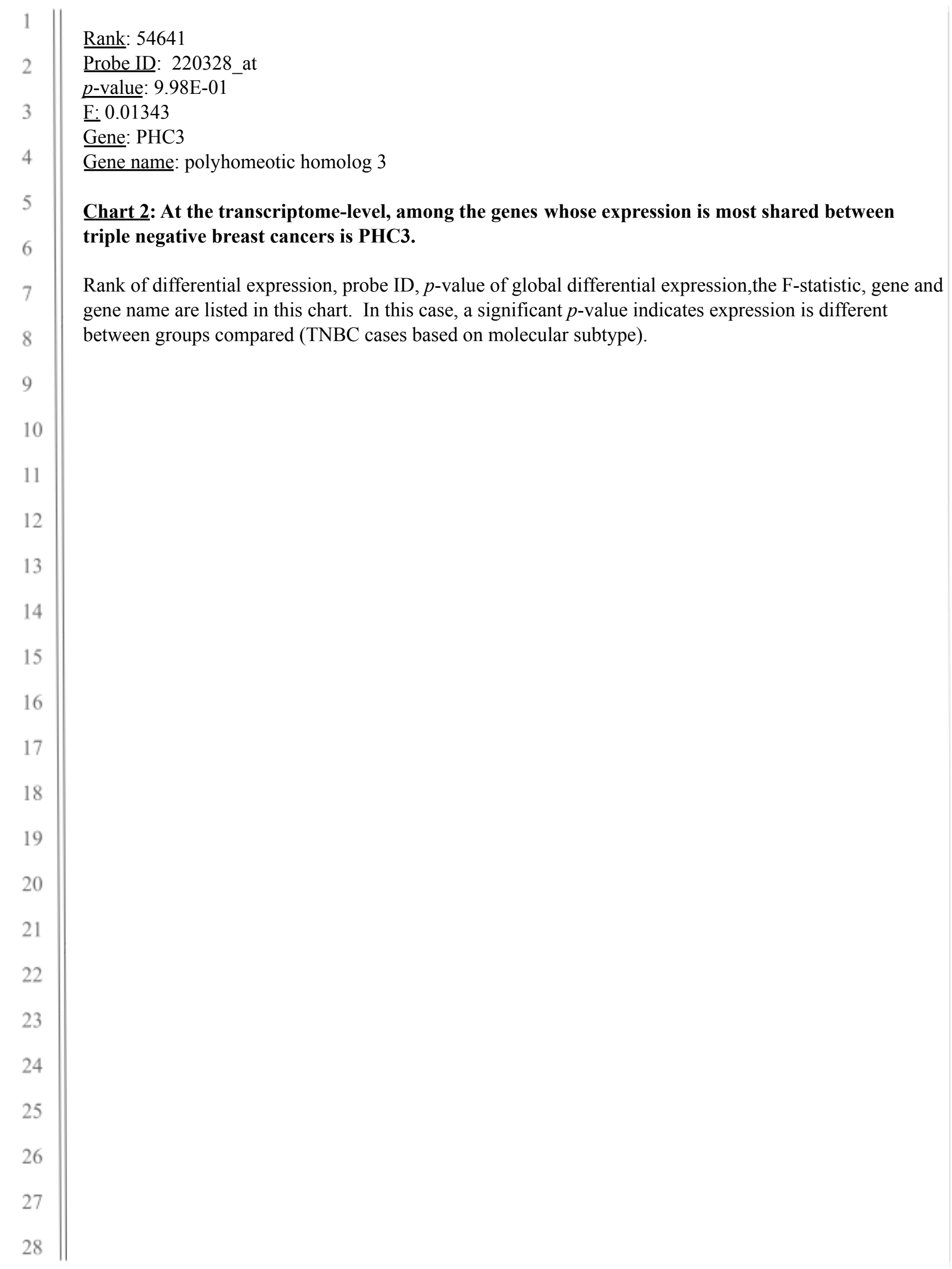




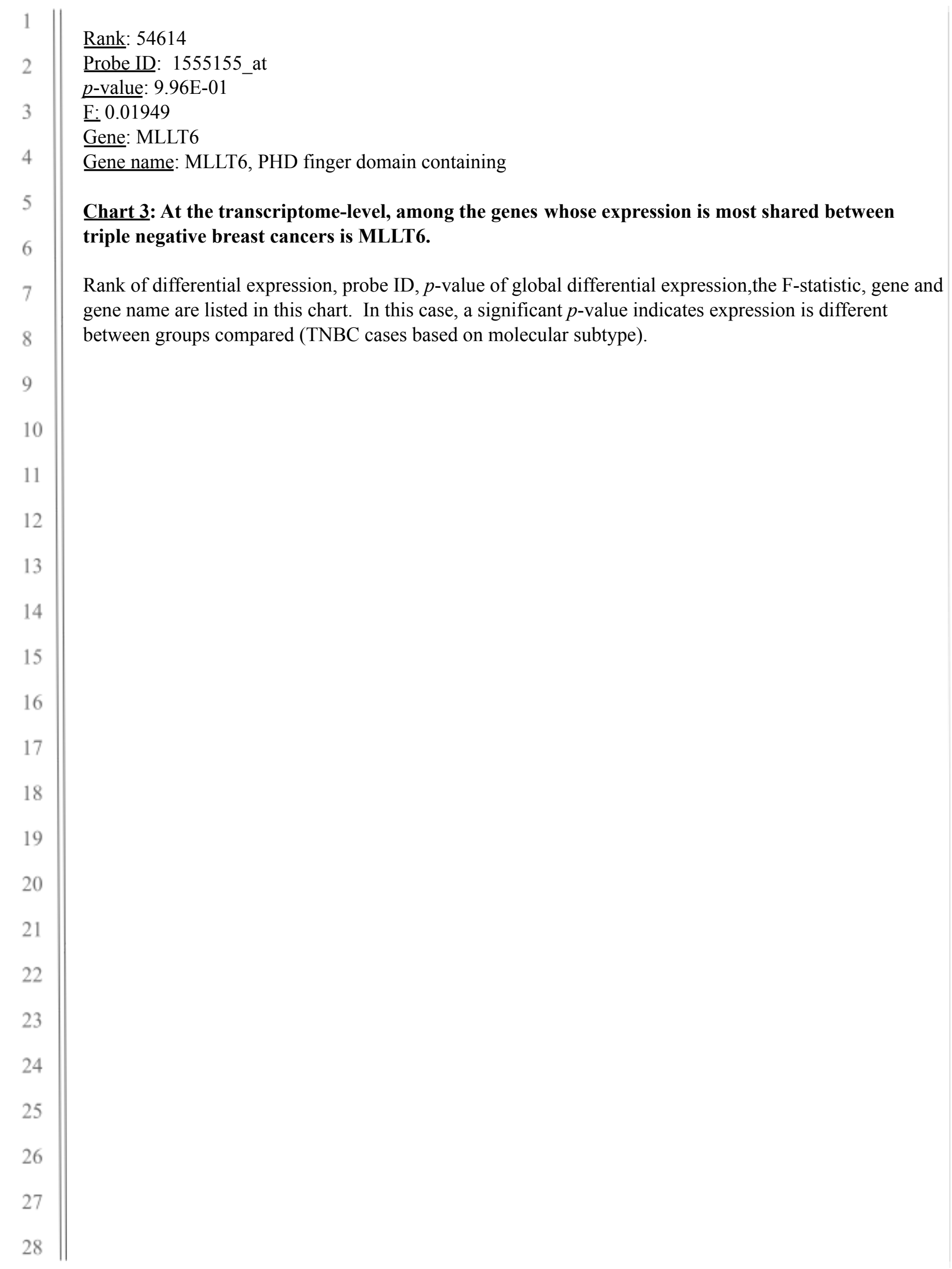




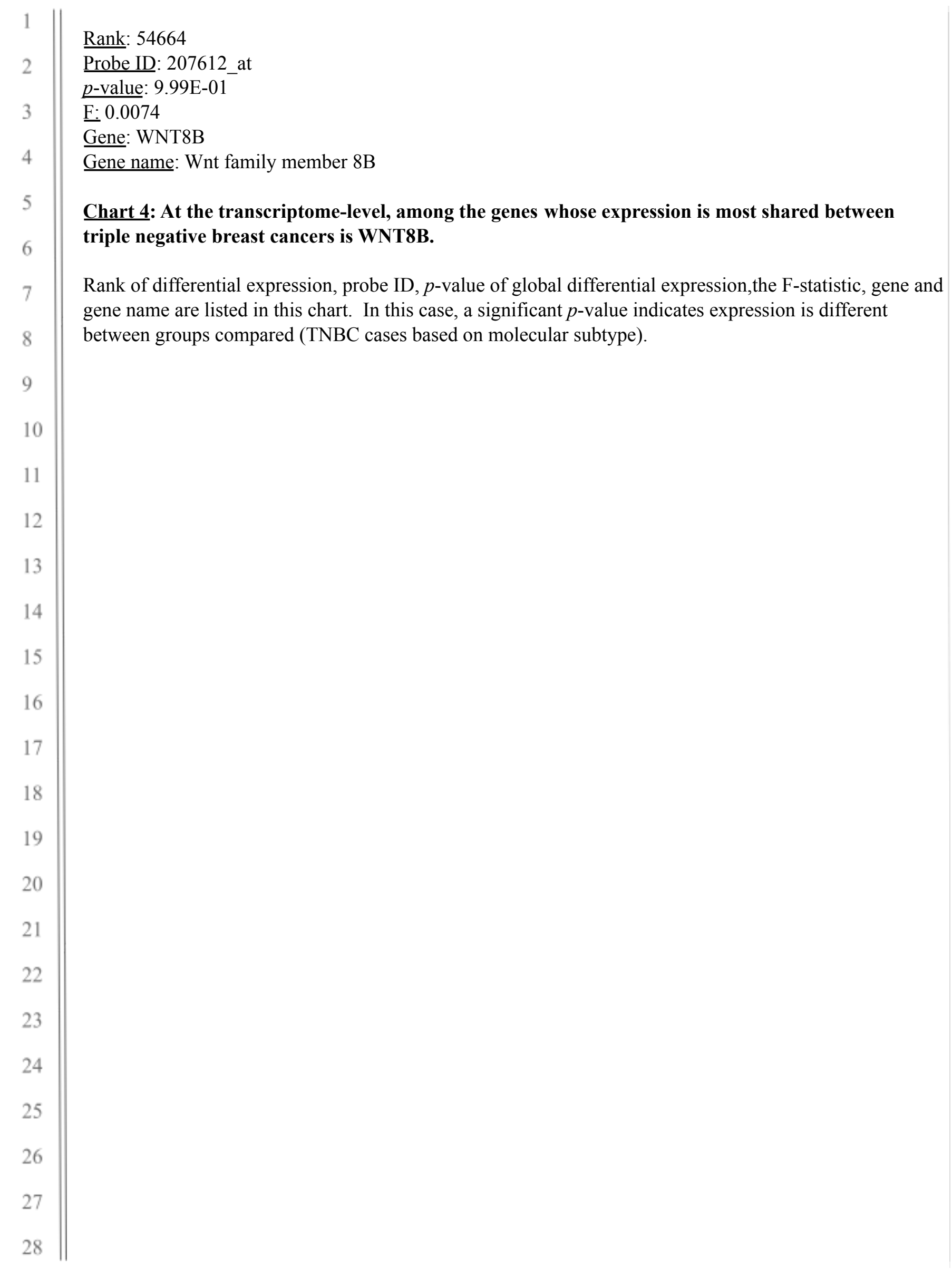




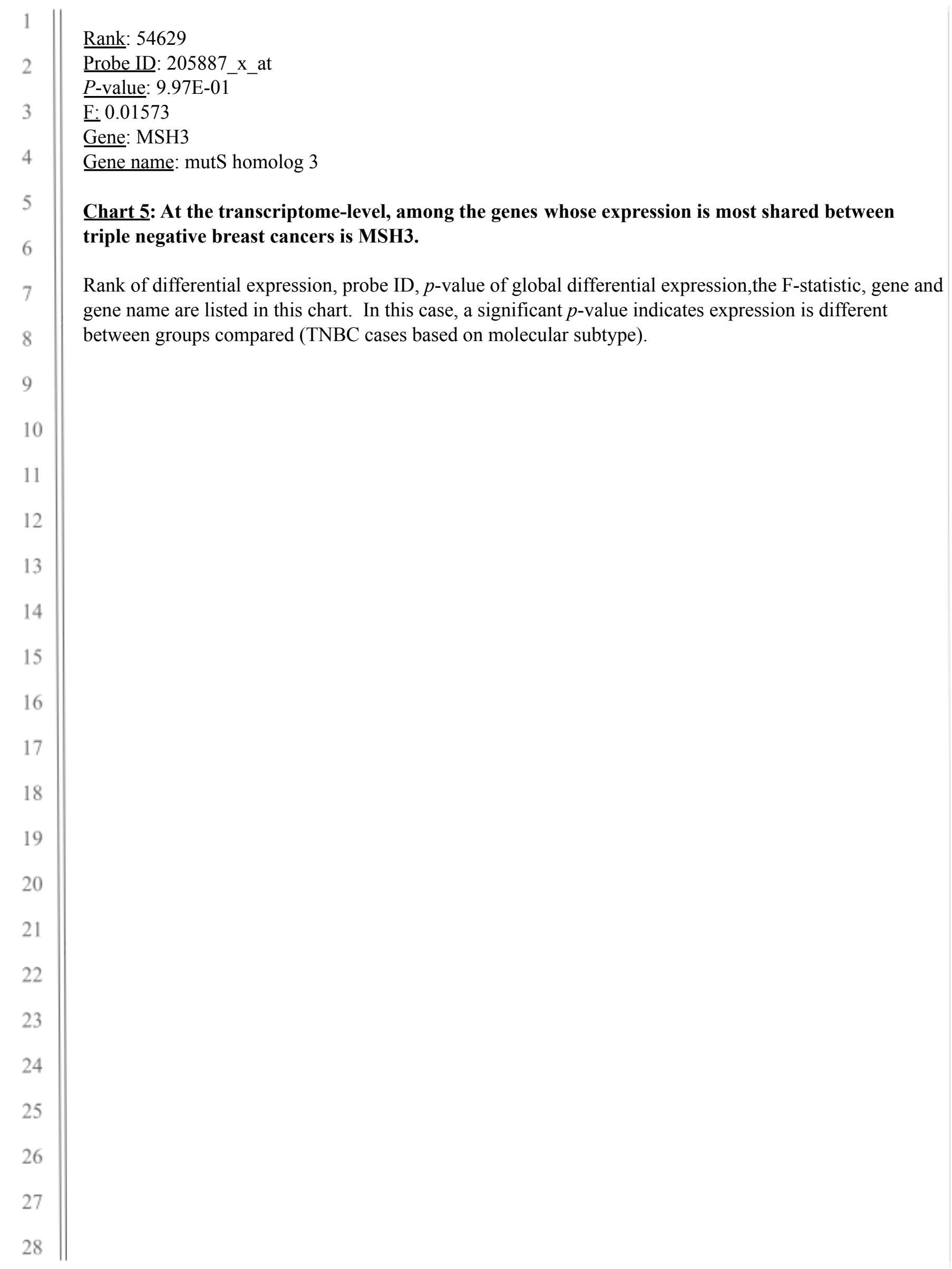




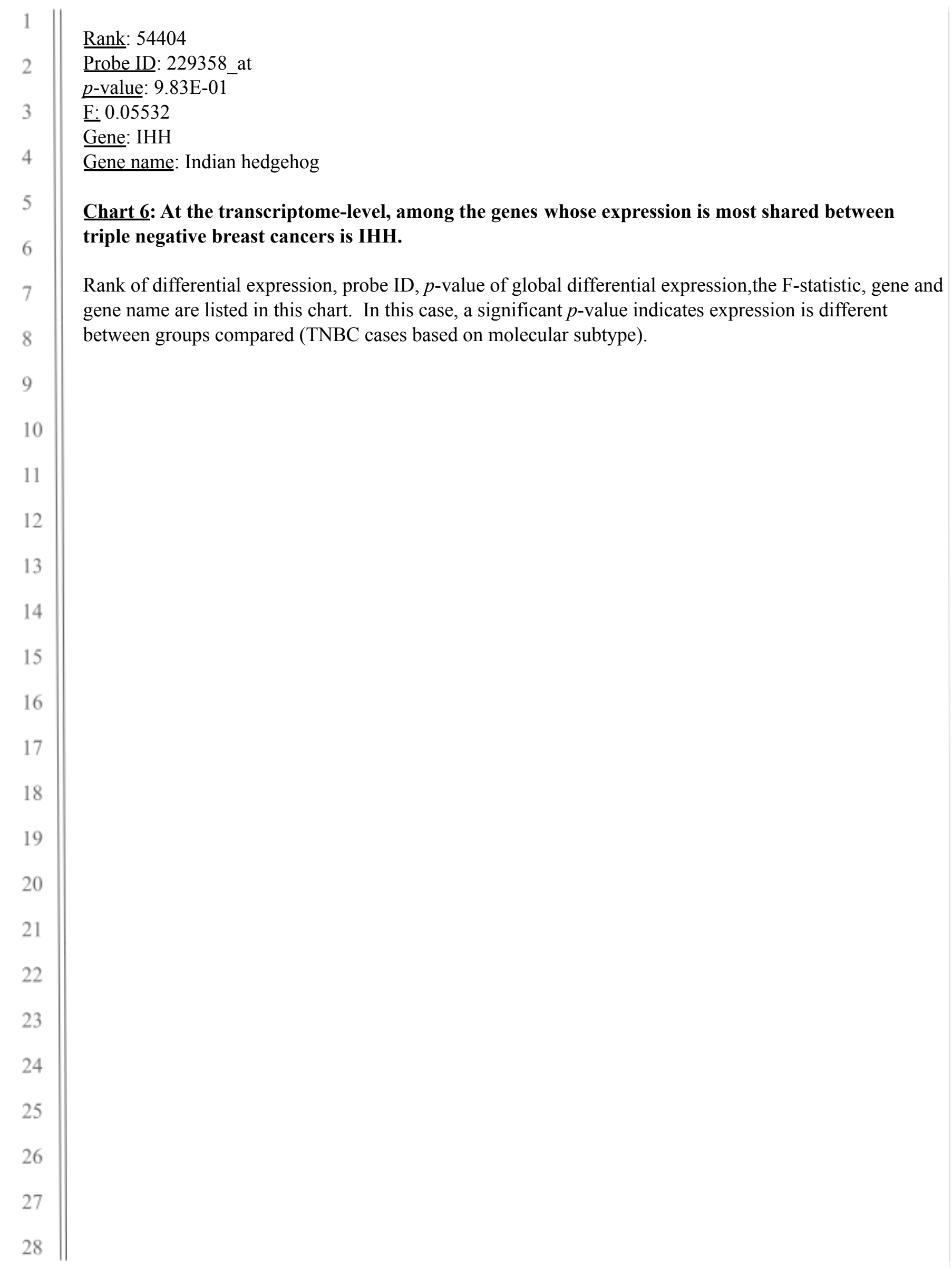




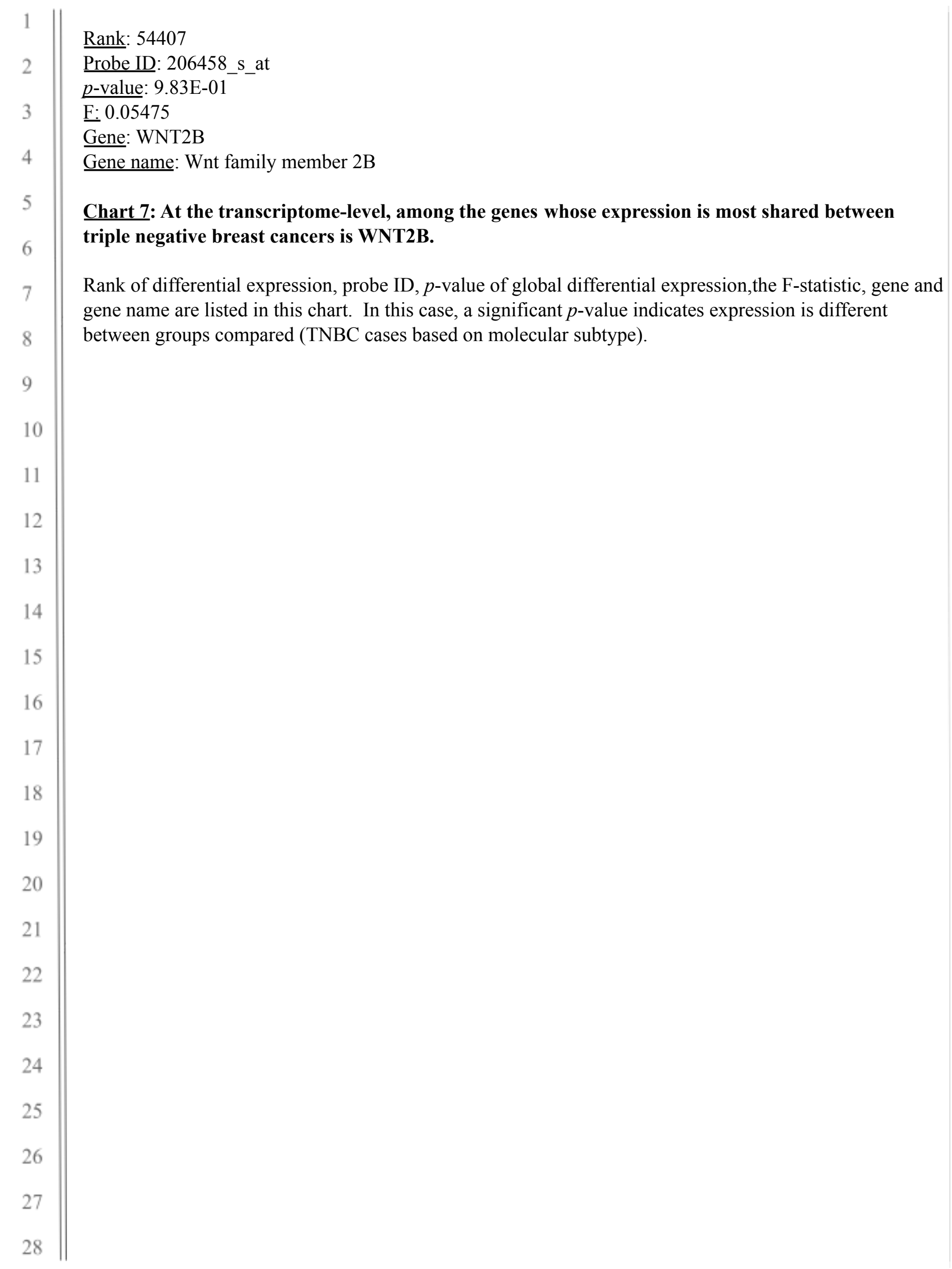



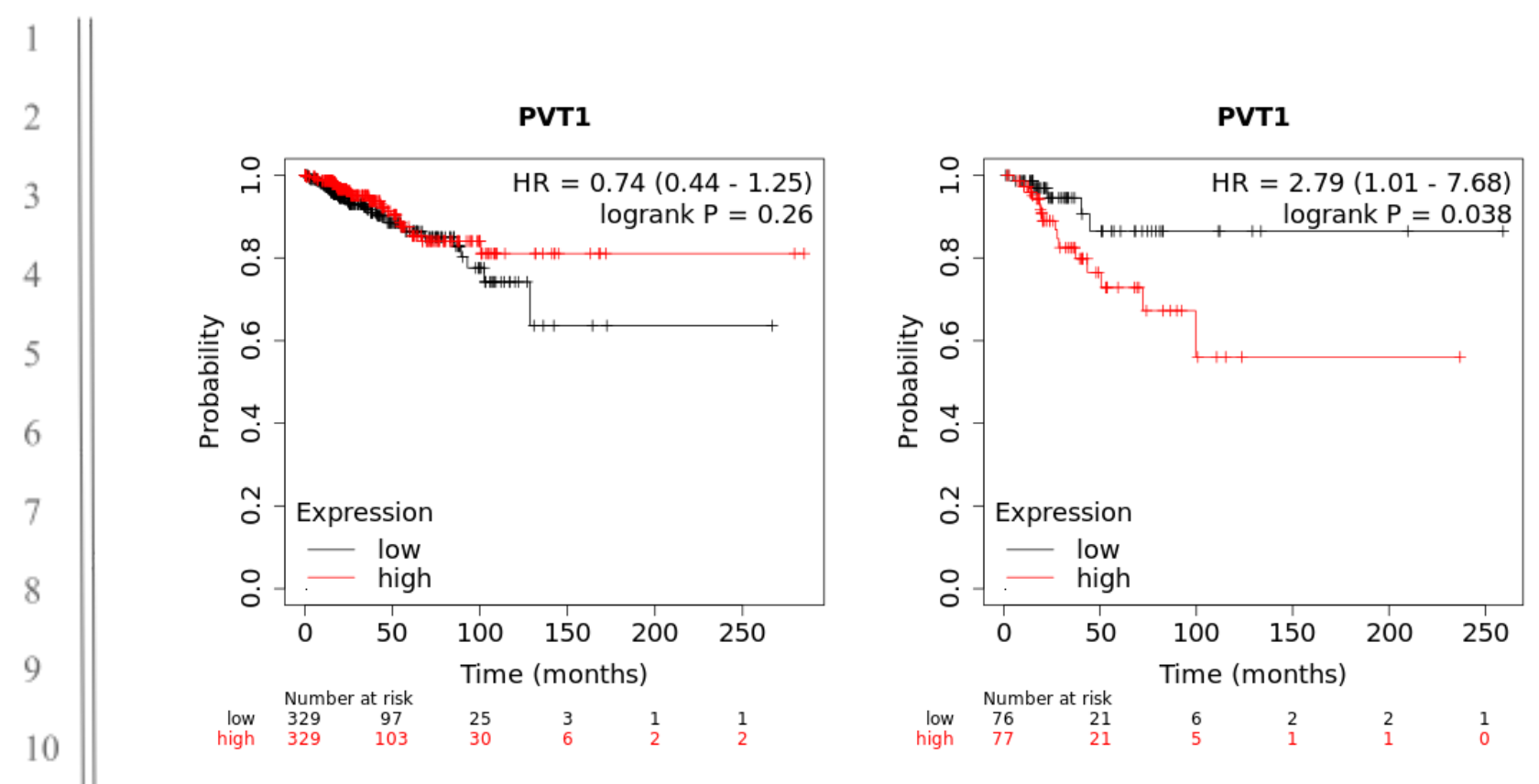

Figure 1: PVT1 expression correlates with recurrence-free survival in black breast cancer patients but not in white breast cancer patients.

Depicted in this Kaplan-Meier plot is the probability of recurrence-free survival (RFS) for $n=1090$ breast cancer patients of white race (left) or of black race (right), based on low or high expression of PVT1 in patient primary tumors. The log rank p-value denoting statistical significance of difference in recurrence-free survival when comparing the two groups, as well as hazard ratio for this comparison is listed above. Listed below is the number of patients at risk (number of patients alive) per interval, after stratification based on PVT1 expression; in the first interval, number at risk is number of patients alive; in each subsequent interval, number at risk is the number at risk less those who have expired or are censored. 

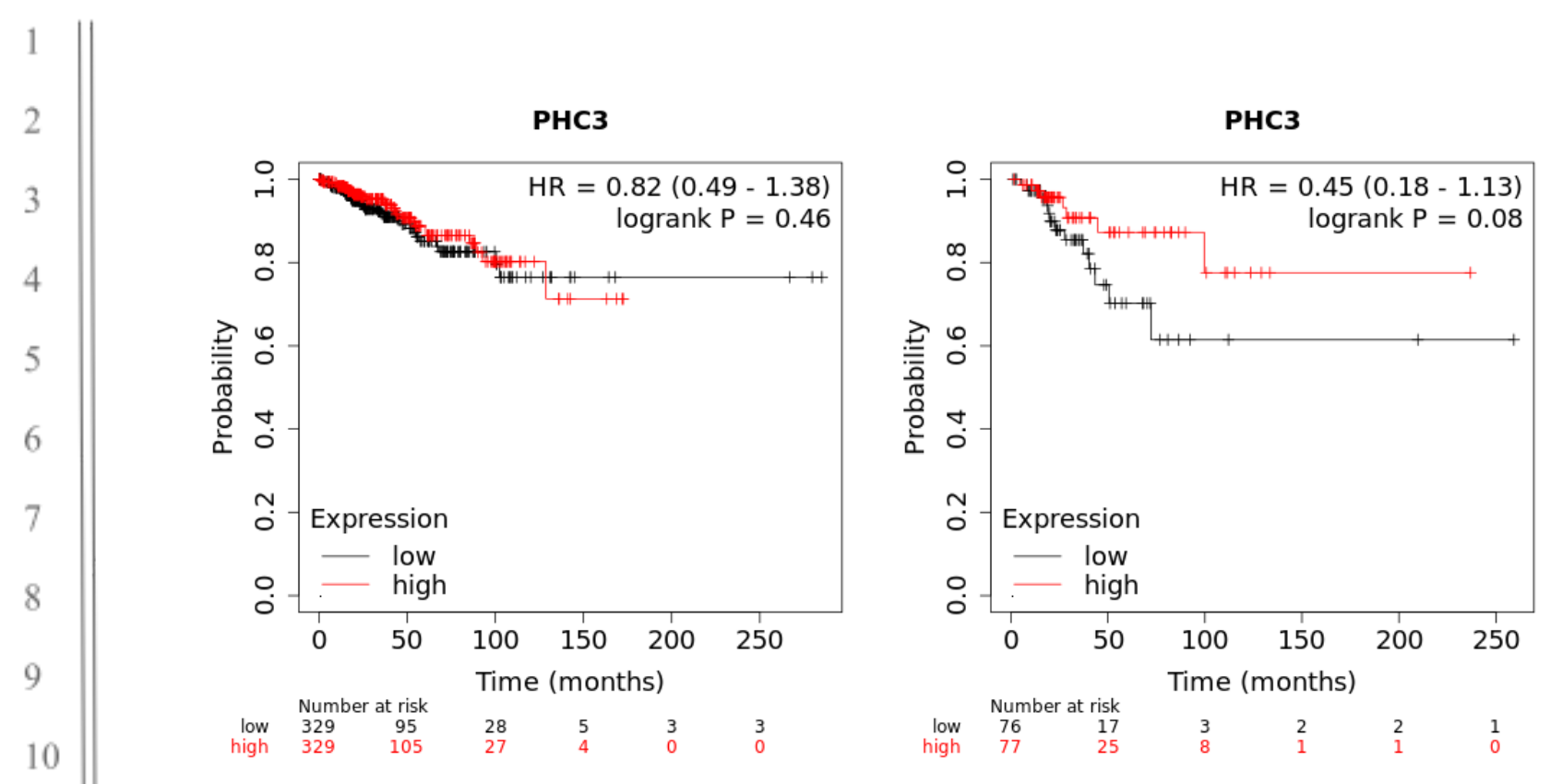

Figure 2: PHC3 expression correlates with recurrence-free survival in black breast cancer patients but not in white breast cancer patients.

Depicted in this Kaplan-Meier plot is the probability of recurrence-free survival (RFS) for $n=1090$ breast cancer patients of white race (left) or of black race (right), based on low or high expression of PHC3 in patient primary tumors. The log rank p-value denoting statistical significance of difference in recurrence-free survival when comparing the two groups, as well as hazard ratio for this comparison is listed above. Listed below is the number of patients at risk (number of patients alive) per interval, after stratification based on PVT1 expression; in the first interval, number at risk is number of patients alive; in each subsequent interval, number at risk is the number at risk less those who have expired or are censored. 


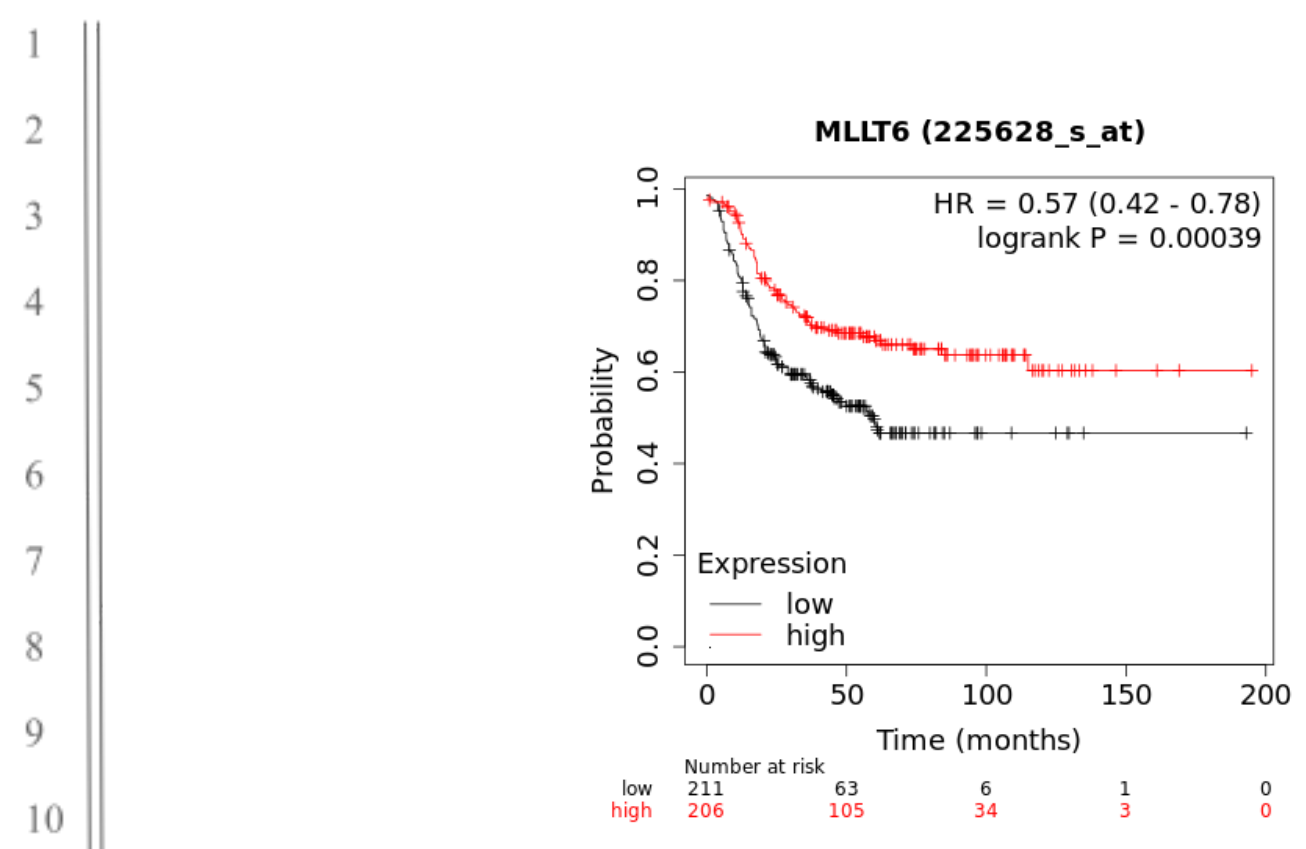

Figure 3: MLLT6 expression correlates with recurrence-free survival in patients with basal subtype cancer.

Depicted in this Kaplan-Meier plot is the probability of recurrence-free survival (RFS) for $n=417$ patients with basal subtype breast cancer stratified into two groups, based on low or high expression of MLLT6 in patient primary tumors. The log rank p-value denoting statistical significance of difference in recurrence-free survival when comparing the two groups, as well as hazard ratio for this comparison is listed above. Listed below is the number of patients at risk (number of patients alive) per interval, after stratification based on MLLT6 expression; in the first interval, number at risk is number of patients alive; in each subsequent interval, number at risk is the number at risk less those who have expired or are censored. 


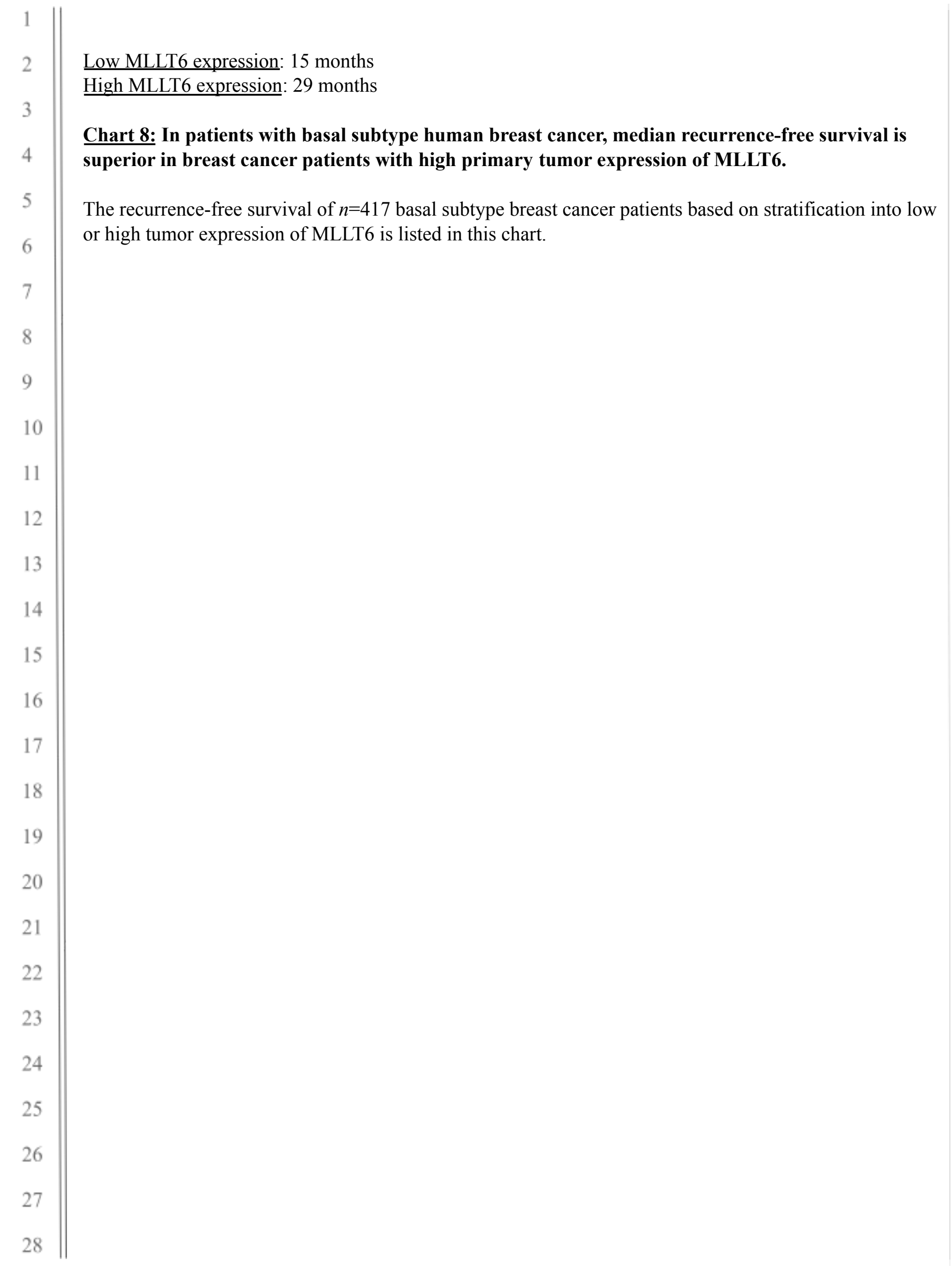




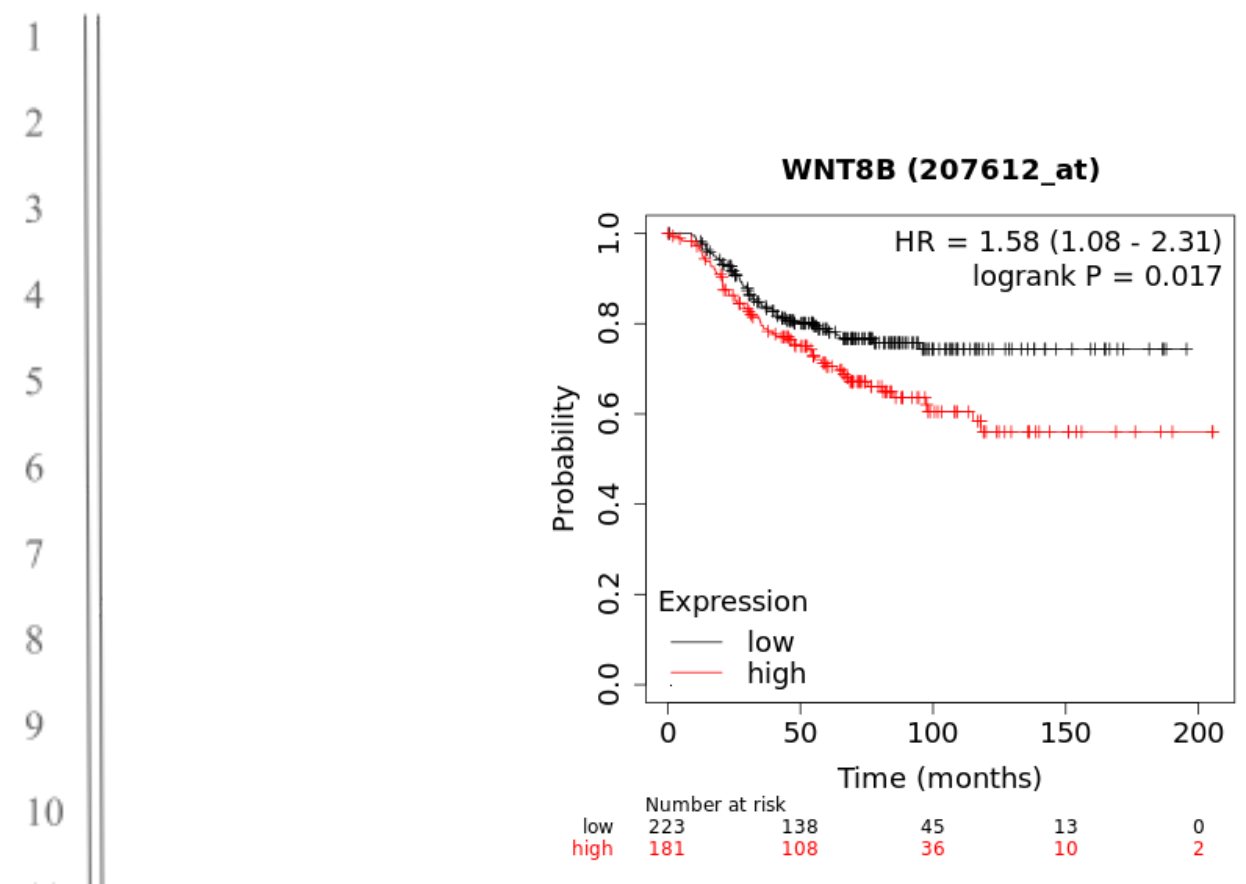

Figure 4: WNT8B expression correlates with overall survival in patients with basal subtype cancer.

Depicted in this Kaplan-Meier plot is the probability of overall survival (OS) for $n=404$ patients with basal subtype breast cancer stratified into two groups, based on low or high expression of WNT8B in patient primary tumors. The log rank p-value denoting statistical significance of difference in recurrence-free survival when comparing the two groups, as well as hazard ratio for this comparison is listed above. Listed below is the number of patients at risk (number of patients alive) per interval, after stratification based on WNT8B expression; in the first interval, number at risk is number of patients alive; in each subsequent interval, number at risk is the number at risk less those who have expired or are censored. 


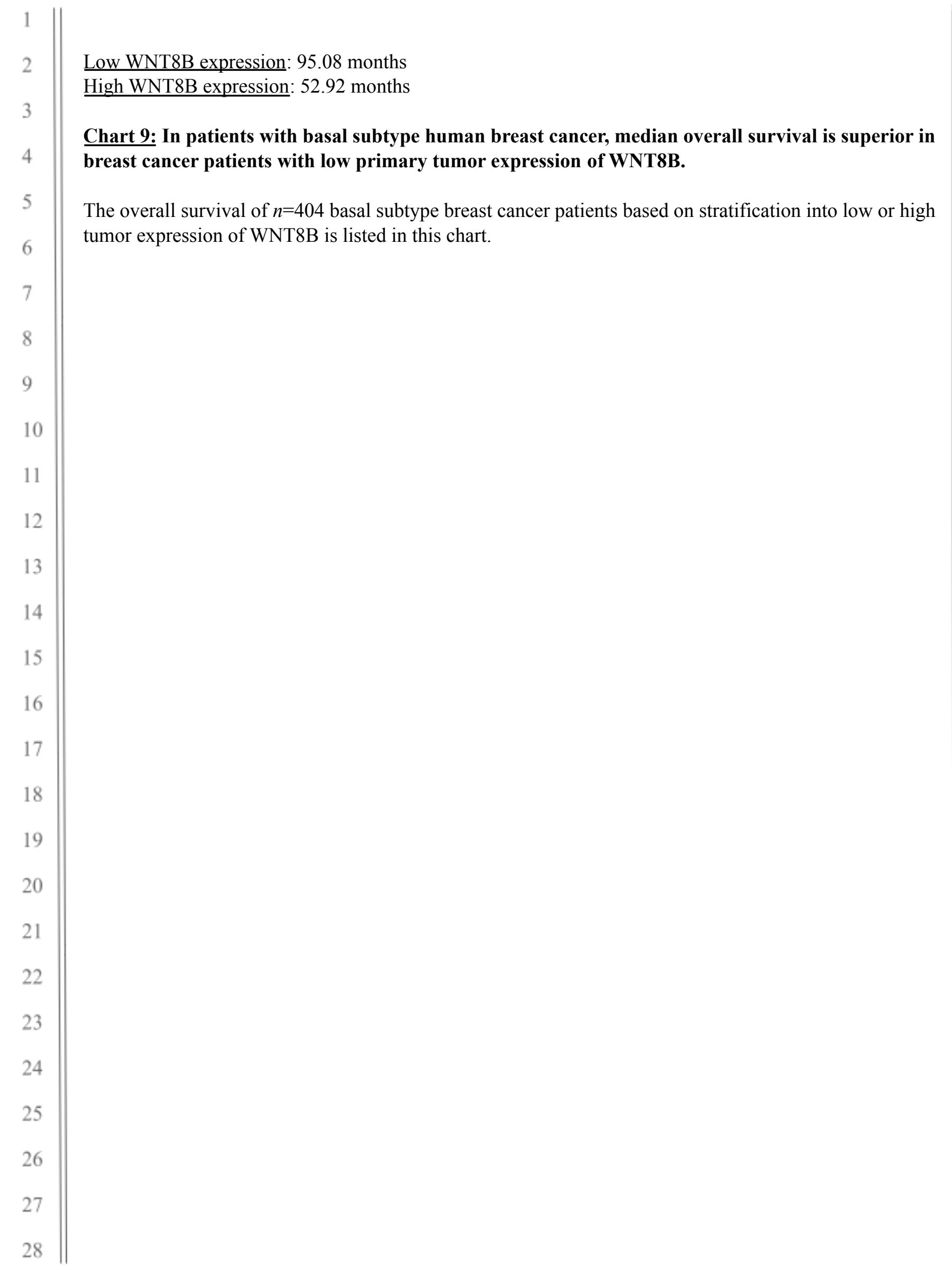




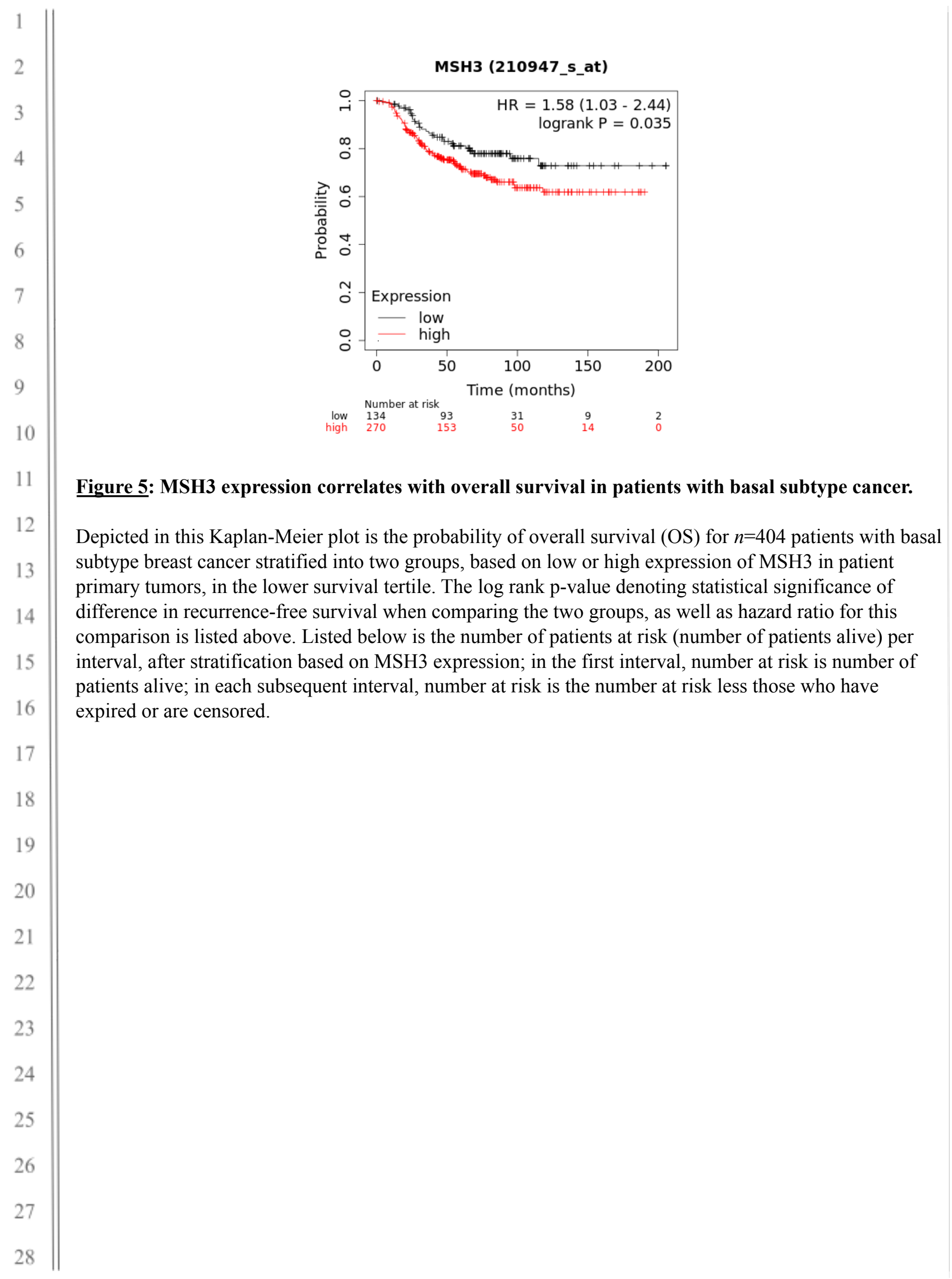




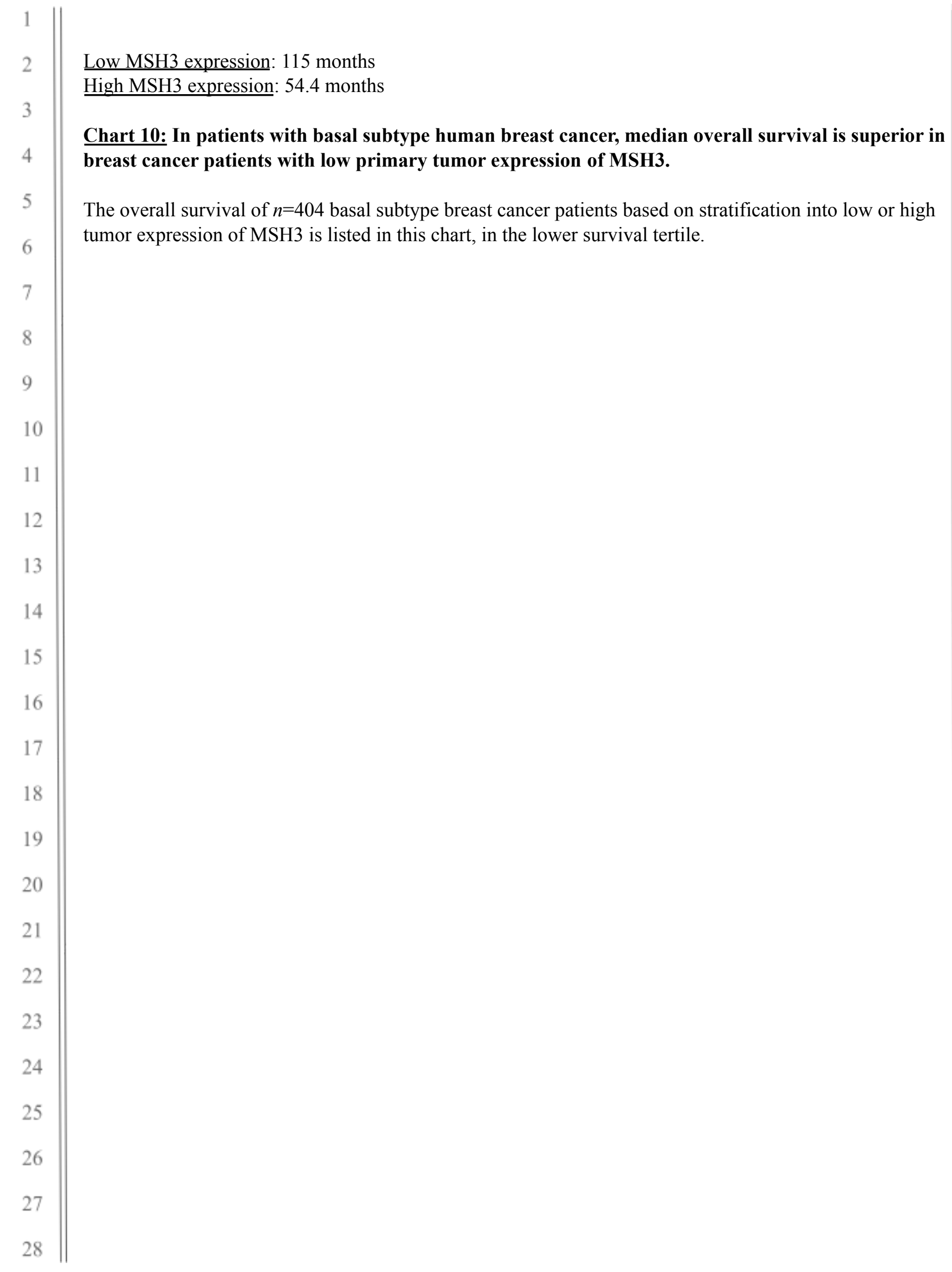




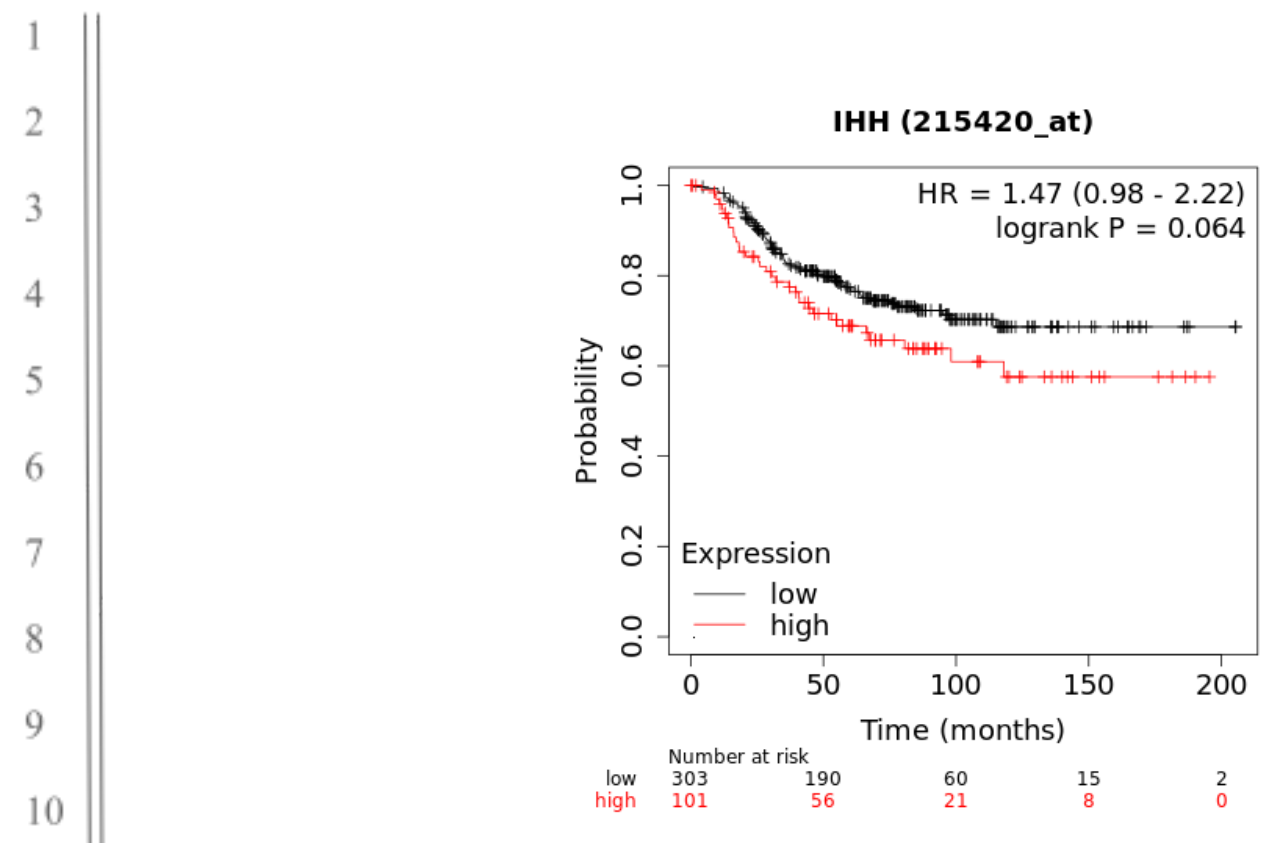

11 Figure 6: IHH expression correlates with overall survival in patients with basal subtype cancer.

Depicted in this Kaplan-Meier plot is the probability of overall survival (OS) for $n=404$ patients with basal subtype breast cancer stratified into two groups, based on low or high expression of IHH in patient primary tumors, in the upper survival quartile. The log rank p-value denoting statistical significance of difference in recurrence-free survival when comparing the two groups, as well as hazard ratio for this comparison is listed above. Listed below is the number of patients at risk (number of patients alive) per interval, after stratification based on IHH expression; in the first interval, number at risk is number of patients alive; in each subsequent interval, number at risk is the number at risk less those who have expired or are censored. 


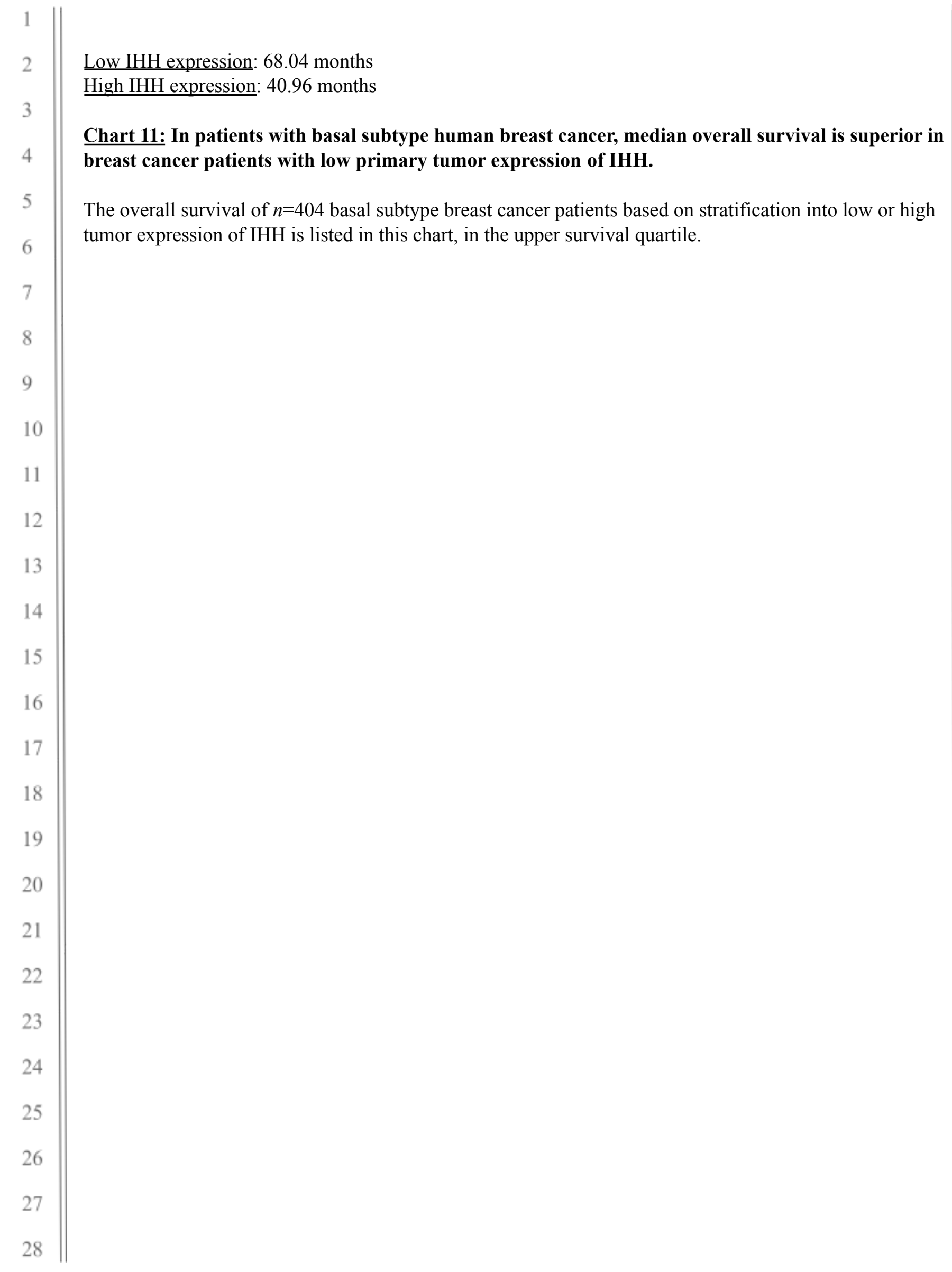




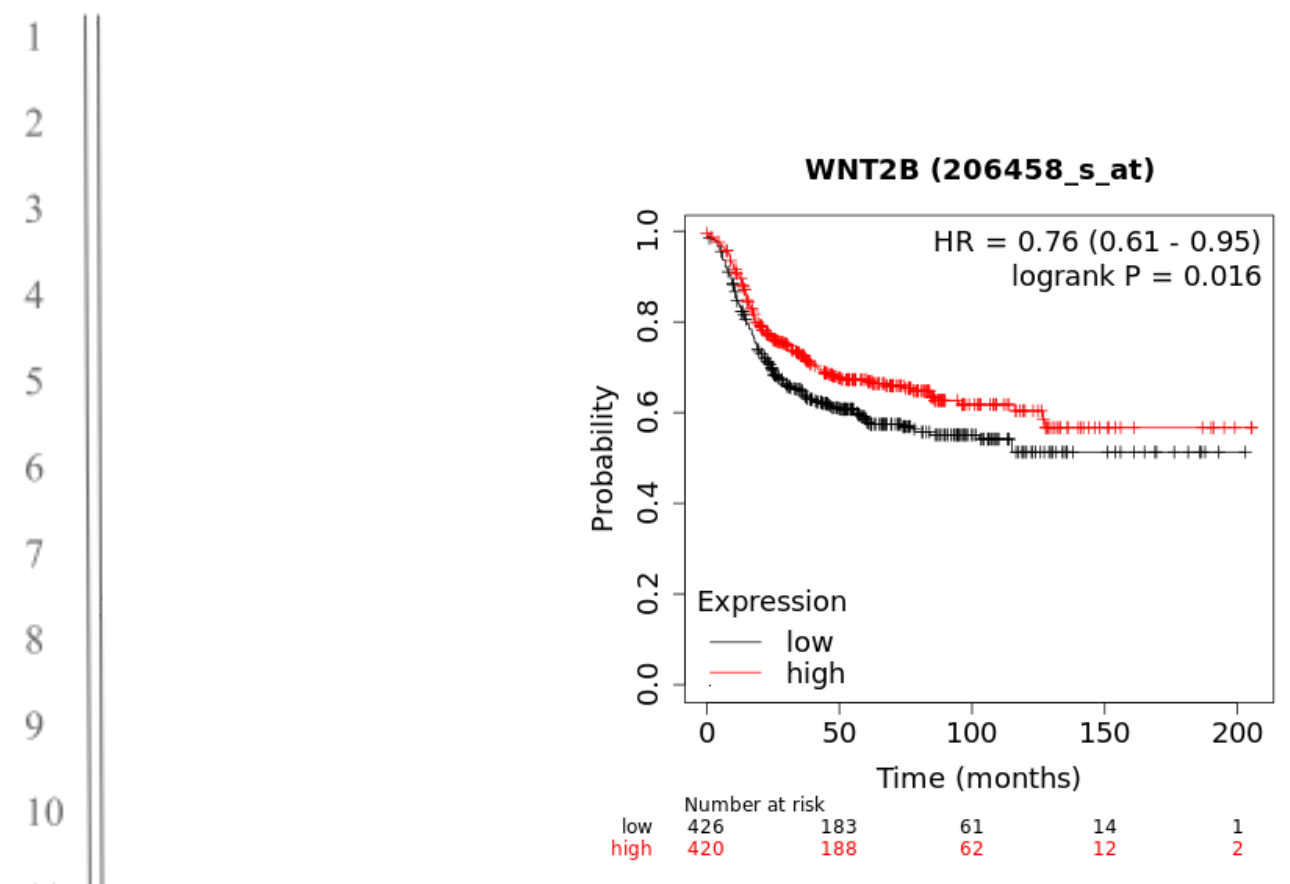

Figure 7: WNT2B expression correlates with recurrence-free survival in patients with basal subtype cancer.

Depicted in this Kaplan-Meier plot is the probability of recurrence-free survival (RFS) for $n=846$ patients with basal subtype breast cancer stratified into two groups, based on low or high expression of WNT2B in patient primary tumors. The log rank p-value denoting statistical significance of difference in recurrence-free survival when comparing the two groups, as well as hazard ratio for this comparison is listed above. Listed below is the number of patients at risk (number of patients alive) per interval, after stratification based on WNT2B expression; in the first interval, number at risk is number of patients alive; in each subsequent interval, number at risk is the number at risk less those who have expired or are censored. 


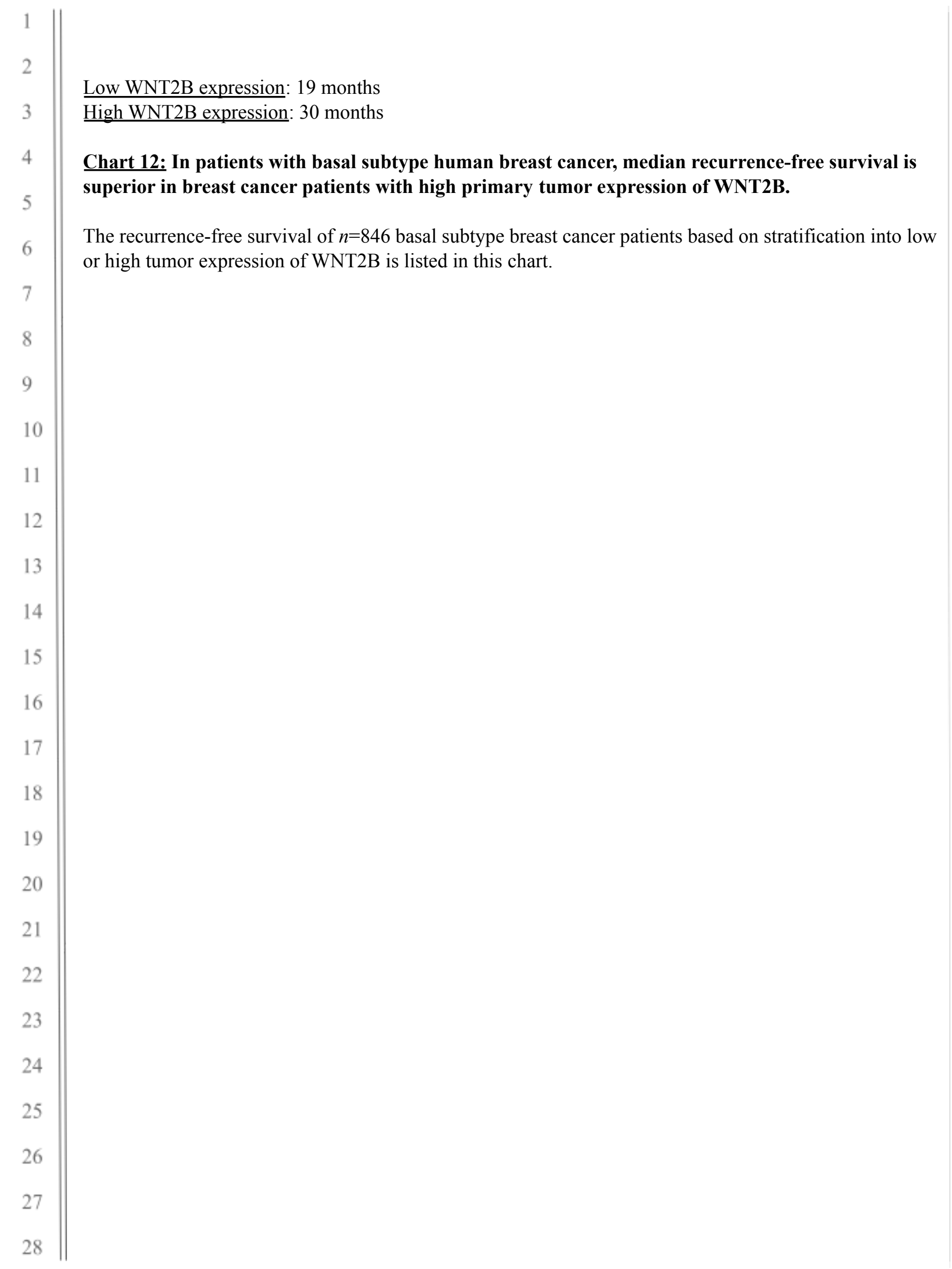

